\title{
Photon Echoes Made Simple $\dagger$
}

\author{
R. BEACH, B. BRODY $\ddagger$ and S. R. HARTMANN
}

Columbia Radiation Laboratory, Department of Physics, Columbia University, New York, NY 10027

Photon Echoes in gases are analyzed via the Billiard Ball Echo Model both in the short and long pulse limit where the "atoms" are represented as spherical and elliptical billiard balls, respectively. Recent experiments demonstrating the elliptical billiard ball shape are presented using intensity data which ranges over close to eleven orders of magnitude.

\section{INTRODUCTION}

In $1950 \mathrm{E}$. L. Hahn observed the coherent rephasing of nuclear magnetic moments in a paramagnetic sample after it had been subjected to two resonant bursts of an oscillating magnetic field. ${ }^{1}$ The rephasing was found to occur at a time after the second excitation pulse equal to the time between the first and second excitations. The rephasing signals were called spin echoes and were beautifully explained by Hahn using what is now known as the vector model. In 1964 Kurnit $e$ al. at Columbia observed the analogous phenomenon in the optical regime. ${ }^{2}$ Termed photon echoes this phenomenon was first seen in a dilute ruby crystal using a pulsed ruby laser. Several years later, in 1968, Patel and Slusher performed an experiment in which photon echoes were generated in a gas. ${ }^{3}$ This observation was important in as much as it demonstrated that echoes could be produced with atoms or molecules which moved many optical wave lengths in the course of producing an echo. A theoretical paper by Scully et al. also published in 1968 provided the analysis appropriate

† This work was supported by the Joint Services Electronics Program (US Army, US Navy, and US Air Force) under Contract No. DAAG29-82-K-0080 and by the Office of Naval Research under Contract No. N00014-78-C-0517.

$\ddagger$ Permanent address: Bard College, Annandale-on-Hudson, NY 12504. 
for a gas and demonstrated that echo generation was possible in samples of non-stationary atoms. ${ }^{4}$ It now seems in fact that the gaseous medium is ideal in as much as a far wider variety of echoes have been produced in gases than in solids. Echo production in a gas seems to be even easier than in a solid. The simplicity of the echo producing mechanisms in a gas until recently has not been matched by a simplicity in analysis. Recently however an unorthodox view has been taken of the effect of coherent radiation pulses on atoms or molecules with a resonant transition. This view has led to the Billiard Ball Echo theory which explains all observed photon echo behavior in gases. ${ }^{5}$ Twopulse, three-pulse, tri-level, Raman, and two-photon echoes are handled with equal ease. The key is atomic recoil. Radiation pulses are comprised of photons and photons carry momentum. In any absorption or emission process momentum must be conserved. The Billiard Ball Echo Model explicitly monitors this momentum conservation and reduces the echo production problem to a problem in kinematics. It works as follows. Laser pulses split atomic wave packets into separate parts corresponding to the states that are coupled by the excitations. These parts then separate because of the recoil momentum. We follow their motion as they move freely between excitation pulses and are again split by successive excitation pulses; the equivalent to coherent rephasing occurs whenever the wave packets of two states that support a radiative moment and that have originated from the same parent collide. This is the essence of the Billiard Ball Echo Model which we now develop in greater detail.

\section{BILLIARD-BALL MODEL}

We begin by considering an atom initially in its ground state and localized at the origin. We irradiate this atom with a laser pulse having wave vector $\mathbf{k}$ and frequency $\omega=\Omega+\hbar k^{2} / 2 \mathrm{~m}$. Here $\hbar \Omega$ is the energy splitting of the $|1\rangle$ (ground) state and $|2\rangle$ (excited) state and $\hbar k^{2} / 2 m$ is the frequency associated with atomic recoil. The state of the atom before it sees the laser pulse is given by

$$
|\Psi(t)\rangle_{\text {before }}=\int d \mathbf{q} A(\mathbf{q}) e^{-\left(\hbar \mathbf{q}^{2} / 2 m\right) t}|\mathbf{q}\rangle|1\rangle
$$

where $|\mathbf{q}\rangle$ is the center-of-mass eigenket of an atom having center-ofmass momentum $\hbar \mathbf{q}$ and $\boldsymbol{A}(\mathbf{q})$ is related to the Maxwellian momentum 
distribution by

$$
|A(\mathbf{q})|^{2}=\left(\pi q^{2}\right)^{-3 / 2} e^{-\mathbf{q}^{2} / q_{0}^{2}}
$$

where

$$
q_{0}=\left(2 m k_{B} T / \hbar^{2}\right)^{1 / 2}
$$

and $m, k_{B}$ and $T$ are the atomic mass, Boltzman's constant and temperature, respectively. After the atom sees the laser pulses we represent its state by

$$
\begin{aligned}
|\Psi(t)\rangle_{\mathrm{after}}= & \cos (\Theta / 2) \int d \mathbf{q} A_{1 \rightarrow 1}(\mathbf{q}) e^{-i\left(\hbar \mathbf{q}^{2} / 2 m\right) t}|\boldsymbol{q}\rangle|1\rangle \\
& +i \sin (\Theta / 2) \int d \mathbf{q} A_{1 \rightarrow 2}(\mathbf{q}) \\
& \times e^{\left.-i(\hbar(\mathbf{q}+\mathbf{k}))^{2} / 2 m\right) t}|\mathbf{q}+\mathbf{k}\rangle e^{-i \Omega t}|2\rangle
\end{aligned}
$$

$A_{1 \rightarrow 1}(\mathbf{q})$ and $A_{1 \rightarrow 2}(\mathbf{q})$ are now generalized momentum distributions and $\Theta$ is the pulse area as defined by

$$
\Theta=\hbar^{-1} p_{21} \int d t E(t)
$$

where $E(t)$ is the envelope of the applied excitation pulse and $p_{21}$ is the radiative moment coupling states $|1\rangle$ and $|2\rangle$. Using the relation

$$
\langle\mathbf{r} \mid \mathbf{q}\rangle=(2 \pi)^{-3 / 2} e^{i \mathbf{r} \cdot \mathbf{q}}
$$

we can write the spatial form of Eq. (4) as

$$
\begin{aligned}
\Psi(\mathbf{r}, t)= & \cos (\Theta / 2)(2 \pi)^{-3 / 2} \int d \mathbf{q} A_{1 \rightarrow 1}(\mathbf{q}) e^{-i\left(\hbar \mathbf{q}^{2} / 2 m\right) t} e^{i \mathbf{q} \cdot \mathbf{r}}|1\rangle \\
& +i \sin (\Theta / 2)(2 \pi)^{-3 / 2} \int d \mathbf{q} A_{1 \rightarrow 2}(\mathbf{q}) \\
& \times e^{-i\left(\hbar(\mathbf{q}+\mathbf{k})^{2} / 2 m\right) t} e^{i(\mathbf{q}+\mathbf{k}) \cdot \mathbf{r}} e^{-i \Omega t}|2\rangle
\end{aligned}
$$

Remembering that $\omega=\Omega+\hbar k^{2} / 2 m$, Eq. (7) can be rewritten as

$$
\begin{aligned}
\Psi(\mathbf{r}, t)= & \cos (\Theta / 2)(2 \pi)^{-3 / 2} \int d \mathbf{q} A_{1 \rightarrow 1}(\mathbf{q}) e^{-i\left(\hbar \mathbf{q}^{2} / 2 m\right) t} e^{i \mathbf{q} \cdot \mathbf{r}}|1\rangle \\
& +i \sin (\Theta / 2)(2 \pi)^{-3 / 2} e^{-i(\omega t-\mathbf{k} \cdot \mathbf{r})} \\
& \times \int d \mathbf{q} A_{1 \rightarrow 2}(\mathbf{q}) e^{-i\left(\hbar \mathbf{q}^{2} / 2 m\right) t} e^{i \mathbf{q} \cdot(\mathbf{r}-(\hbar \mathbf{k} / m) t)}|2\rangle
\end{aligned}
$$


Since we are ultimately concerned with the radiated electric field we will drop the wave packet spreading factors in Eq. (8). That these factors can be dropped when calculating the coherently radiated field is proven in Refs. 5 and 6 . This leaves

$$
\begin{aligned}
\Psi(\mathbf{r}, t)= & \cos (\Theta / 2)(2 \pi)^{-3 / 2} \int d \mathbf{q} A_{1 \rightarrow 1}(\mathbf{q}) e^{i \mathbf{q} \cdot \mathbf{r}}|1\rangle \\
& +i \sin (\Theta / 2)(2 \pi)^{-3 / 2} e^{-i(\omega t-\mathbf{k} \cdot \mathbf{r})} \int d \mathbf{q} \\
& \times A_{1 \rightarrow 2}(\mathbf{q}) e^{i \mathbf{q} \cdot(\mathbf{r}-(\hbar \mathbf{k} / m) t)}|2\rangle
\end{aligned}
$$

Defining the Billiard-ball functions

$$
F_{1 \rightarrow 1}(\mathbf{r})=(2 \pi)^{-3 / 2} \int d \mathbf{q} A_{1 \rightarrow 1}(\mathbf{q}) e^{i \mathbf{q} \cdot \mathbf{r}}
$$

and

$$
F_{1 \rightarrow 2}(\mathbf{r})=(2 \pi)^{-3 / 2} \int d \mathrm{q} A_{1 \rightarrow 2}(\mathbf{q}) e^{i \mathbf{q} \cdot \mathbf{r}}
$$

we can write Eq. (9) as

$$
\begin{aligned}
\Psi(\mathbf{r}, t)= & \cos (\Theta / 2) F_{1 \rightarrow 1}(\mathbf{r})|1\rangle \\
& +i \sin (\Theta / 2) e^{-i(\omega t-\mathbf{k} \cdot \mathbf{r})} F_{1 \rightarrow 2}(\mathbf{r}-(\hbar \mathbf{k} / m) t)|2\rangle
\end{aligned}
$$

In the short pulse limit $F_{1 \rightarrow 1}(\mathbf{r})=F_{1 \rightarrow 2}(\mathbf{r})=F(\mathbf{r})$ where

$$
F(\mathbf{r})=\left(q_{0}^{2} / \pi\right)^{3 / 4} e^{-q z^{2} / 2}
$$

This is the case treated in Ref. 5 where all wavepackets are spherical. In Ref. 6 the short pulse restriction is lifted and the laser linewidth does not necessarily cover the Doppler broadened transition line uniformly. In this case $F_{1 \rightarrow 1}(\mathbf{r})$ and $F_{1 \rightarrow 2}(\mathbf{r})$ are given by

$$
F_{1 \rightarrow 1}(\mathbf{r})=\left(q_{0}^{2} / \pi\right)^{1 / 2} e^{-q 2 r_{\perp}^{2} / 2} f_{1 \rightarrow 1}\left(r_{k}\right)
$$

and

$$
F_{1 \rightarrow 2}(\mathbf{r})=\left(q_{0}^{2} / \pi\right)^{1 / 2} e^{-q 2 r_{\perp}^{2} / 2} f_{1 \rightarrow 2}\left(r_{k}\right)
$$

We have decomposed $r$ according to

$$
\mathbf{r}=r_{\perp} \hat{\perp}+r_{k} \hat{k}
$$


where $\hat{k}$ is in the direction of the applied laser pulse and $\hat{\perp}$ is a generalized unit vector perpendicular to the applied laser pulse. In Ref. 6 it is shown that sub-Doppler width laser pulses generally give rise to Billiard-ball functions that are modulated in the direction of the applied laser pulse. More specifically, in the small pulse area limit with a gaussian shaped electric field given by

$$
\varepsilon(t)=E_{t=0} e^{-\left(t / \tau_{1 / e}\right)^{2 / 2}} \cos \omega t
$$

$f_{1 \rightarrow 1}\left(\mathbf{r}_{k}\right)$ and $f_{1 \rightarrow 2}\left(\mathbf{r}_{k}\right)$ are given by

$$
f_{1 \rightarrow 1}\left(r_{k}\right)=\left(q_{0}^{2} / \pi\right)^{1 / 4} e^{-q_{0}^{2} r_{k}^{2} / 2}
$$

and

$$
f_{1 \rightarrow 2}\left(r_{k}\right)=\left(\left(q_{00}^{2} / q_{0}\right)^{2} / \pi\right)^{1 / 4} e^{-q z_{0} r_{k} / 2}
$$

where

$$
\left(1 / q_{00}\right)^{2}=\left(1 / q_{0}\right)^{2}+\left(\hbar k \tau_{1 / e} / m\right)^{2}
$$

In this case the excited state represented by $F_{1 \rightarrow 2}(\mathbf{r})$ is ellipsoidal, being elongated in the direction of the applied laser pulse and having a length-to-width ratio of

$$
L / W=q_{0} / q_{00}
$$

In the extreme long pulse limit this ratio reduces to

$$
L / W=\tau_{1 / e} / \tau_{D}
$$

where $\tau_{D}$ is the Doppler dephasing time defined by

$$
\tau_{D}=m / q_{0} \hbar k
$$

Eq. (12) is valid only after the pulse has passed the atom. It can therefore not be used to calculate the free decay signal at very short times since for at least the beginning of the free decay the excitation pulse is still present. However, in photon echo experiments the excitation pulse separation is usually long compared to the pulse width. Therefore restricting the use of Eq. (12) to times after the passage of the excitation pulses is of no consequence in the analysis of echo experiments. 


\section{TWO-PULSE ECHOES}

The simplicity that is brought to echo analysis by the Billiard-ball model is best seen with an example. Here we consider an ordinary two-pulse echo experiment performed with short collinear excitation pulses having wave vectors $\mathbf{k}$. We apply pulse 1 and 2 at times 0 and $\tau$, respectively. As shown in Ref. 5 it suffices to consider a single atom when calculating the radiated echo signal since all atoms contribute in an identical fashion.

Just prior to the arrival of the first excitation pulse the state of an atom that is localized at the origin and its ground state is given by

$$
\Psi\left(\mathbf{r}, t=0_{-}\right)=F(\mathbf{r})|1\rangle
$$

After the passage of the first excitation pulse, which we assume has area $\pi / 2$, but before the arrival of the second, the state of the system is given by

$$
\Psi(\mathbf{r}, t)=\left(1 / 2^{1 / 2}\right) F(\mathbf{r})|1\rangle+\left(i / 2^{1 / 2}\right) F(\mathbf{r}-(\hbar \mathbf{k} / m) t) e^{-i(\omega t-\mathbf{k} \cdot \mathbf{r})}|2\rangle
$$

If the second excitation pulse has area $\pi$ then the state of the system after its passage is given by

$$
\begin{aligned}
\Psi(\mathbf{r}, t)= & \left(i / 2^{1 / 2}\right) F(\mathbf{r}-(\hbar \mathbf{k} / m)(t-\tau)) e^{-i(\omega(t-\tau)-\mathbf{k} \cdot \mathbf{r})}|2\rangle \\
& -\left(1 / 2^{1 / 2}\right) F(\mathbf{r}-(\hbar \mathbf{k} / m) \tau) e^{-i \omega \tau}|1\rangle
\end{aligned}
$$

In Ref. 5 it is shown that the radiated electric field is proportional to the matrix element,

$$
\int d \mathbf{r} \Psi^{*}(\mathbf{r}, t) e^{i \mathbf{k} \cdot \mathbf{r}} \hat{p} \Psi(\mathbf{r}, t)
$$

where $\hat{p}$ is the dipole moment operator. If we retain just those terms that contribute to the macroscopically radiated electric field we are left with the radiated field being proportional to the overlap integral,

$$
\int d \mathbf{r} F^{*}(\mathbf{r}-(\hbar \mathbf{k} / m)(t-\tau)) F(\mathbf{r}-(\hbar \mathbf{k} / m) \tau)
$$

This is the central result of the Billiard-ball echo model. If we make the substitution

$$
\mathbf{u}=\mathbf{r}-(\hbar \mathbf{k} / m) \tau
$$


in Eq. (28) we have the radiated field being proportional to

$$
\int d \mathbf{u} F^{*}(\mathbf{u}-(\hbar \mathbf{k} / m)(t-2 \tau)) F(\mathbf{u})
$$

Since the Billiard-ball function $F$ is peaked where its argument is zero we have two criteria for emission of an echo. First, $\mathbf{u}$ must be zero to make the second term in the integral in Eq. (30) large. Then, $\mathbf{u}-(\hbar \mathbf{k} / m)(t-2 \tau)$ must be zero to make the first term in the integral in Eq. (30) large. Since we have already required $\mathbf{u}=\mathbf{0}$ in the first condition the second condition is equivalent to requiring that $(\hbar \mathbf{k} / m)(t-2 \tau)$ be zero for the emission of an echo. This happens at time $t=2 \tau$, the same photon echoes are experimentally observed.

There are two general criteria for the emission of an echo, as illustrated in the previous example. First, the billiard ball functions representing two states that support a radiative moment must come into overlap. Second, the momentum difference of these two states must correspond to the momentum associated with a photon emitted by a transition between the two states under consideration.

In the previous example if we had used long excitation pulses rather than short ones, the only difference would have been that the Billiardball functions $F_{1 \rightarrow 1}(\mathbf{r})$ and $F_{1 \rightarrow 2}(\mathbf{r})$ would not have been the same. As shown in Ref. 6 and already mentioned previously, the billiardballs would become elongated in the direction of the applied excitation pulses. Physically, this means the wave packets representing the ground and excited states would maintain overlap for a longer period of time leading to an echo signal having greater temporal width. However, the basic recoil analysis of the experiment would remain unchanged.

We now demonstrate the utility of the Billiard-ball echo model by analyzing several specific cases. For convenience we assume all pulses to be so short as to uniformly excite the entire Doppler broadened sample. This allows us to use spherical billiard-balls throughout, making the presentation cleaner and easier to follow.

\section{TRI-LEVEL ECHOES}

The tri-level echo has been used very successfully to study perturber induced broadening of forbidden ground-to-Rydberg-state 
transitions. ${ }^{7,8,9}$ We now analyze such a tri-level echo experiment using the billiard-ball model.

We consider a three level atom with level diagram as presented in Figure 1a. The energy splitting of the ground and first excited state is $\hbar \Omega_{1}$ and this is assumed to be less than $\hbar \Omega_{2}$, the energy splitting of the first and second excited states. We also assume that the ground and first excited states are coupled by a radiative moment and similarly for the first and second excited states. The sequences of events leading

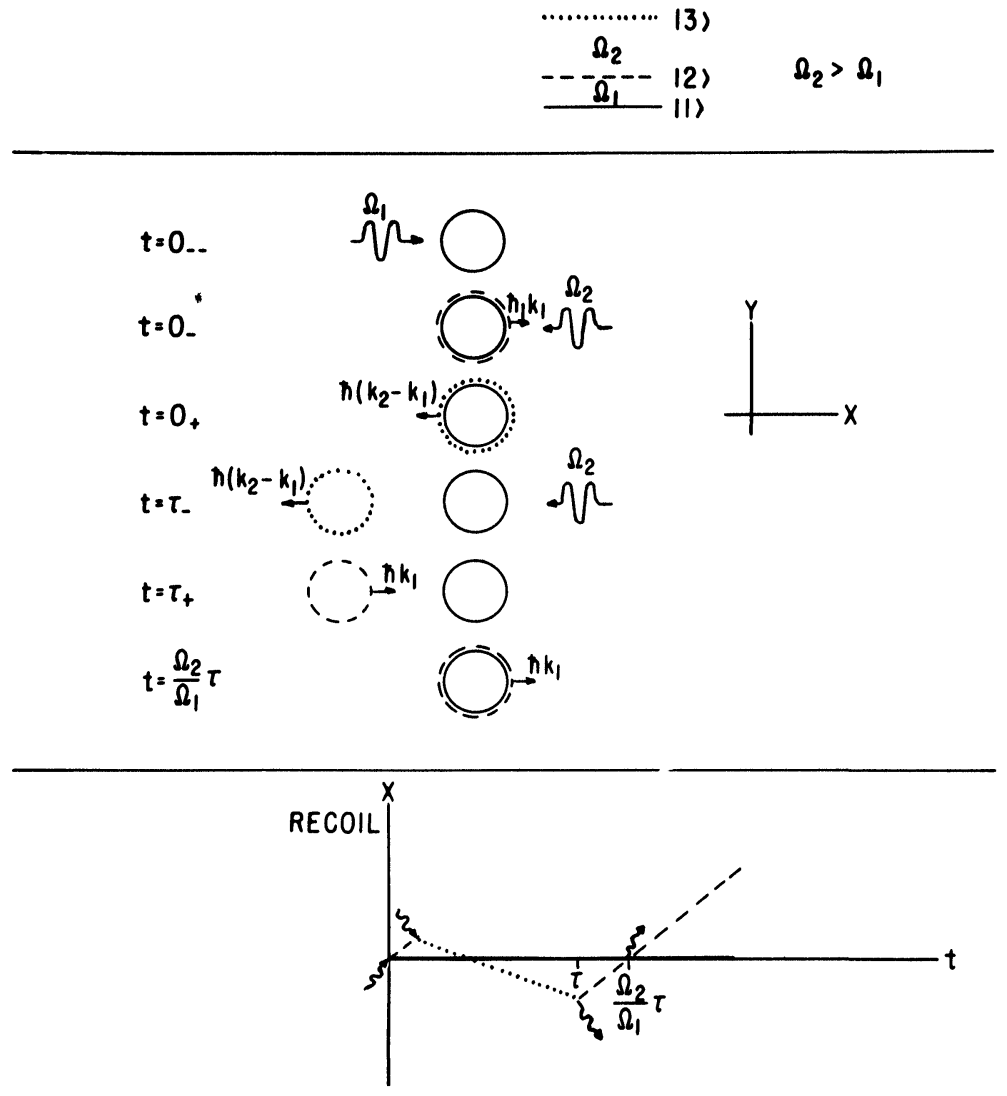

FIGURE 1 (a) Level diagram of the atoms used to generate a tri-level echo. (b) The sequence of events leading to the tri-level echo. Solid line circles denote state $|1\rangle$, dashed line circles denote state $|2\rangle$, and dotted line circles denote state $|3\rangle$. (c) Recoil diagram showing the kinematics of the tri-level echo. 
to the tri-level echo is presented pictorially in Figure $1 \mathrm{~b}$. At $t=0_{--}$ we show an atom in its ground state, as represented by the solid-linecircle, and a laser pulse of frequency $\Omega_{1}$ approaching from the left. We assume this laser pulse has area $\pi / 2$ so that after its passage the atom is in a 50-50 superposition of the ground and first excited state. We show this in the band labelled $t=0_{-}$. The first excited state is represented by the dashed-line circle and is recoiling to the right with the momentum of the absorbed photon, $\hbar k_{1}$. In this band we also show a second laser pulse approaching in the opposite direction. This laser pulse has frequency $\Omega_{2}$ and is assumed to have area $\pi$ : it will promote atoms from the first excited state to the second excited state with $100 \%$ probability. We show the state of the system after the passage of this pulse in the band labelled $t=0_{+}$. The first excited state has been completely transformed to the second excited state, which we represent by the dotted-line circle. Now the second excited state recoils toward the left with momentum $\hbar\left(k_{2}-k_{1}\right)$, the vector momentum of the absorbed photon from pulse two plus one. We let the system develop for time $\tau$ and then send a laser pulse in from the right again having frequency $\Omega_{2}$ and pulse area $\pi$. The situation is depicted in the band labelled $t=\tau_{-}$. The second excited state, recoiling with momentum $\hbar\left(k_{2}-k_{1}\right)$ toward the left moves away from the (at rest) ground state. This third excitation pulse does not interact with the ground state because there is no allowed resonant transition it can couple the ground state to. However it stimulates the second excited state to emit a photon having frequency $\Omega_{2}$, toward the left. Therefore the effect of this excitation pulse is to transform the second excited state completely into the first excited state. Calling $p_{r}$ the recoil momentum of this newly generated first excited state, conservation of momentum requires $p_{r}$ to satisfy

$$
p_{r}-\hbar k_{2}=-\hbar\left(k_{2}-k_{1}\right)
$$

On the left of Eq. (31) we have written the final momentum of the system, which equals the vector sum of the recoil momentum of the first excited state $p_{r}$ and the momentum of the emitted photon, $\hbar k_{2}$. The initial momentum on the right of Eq. (31) is the recoil momentum the second excited state had before the passage of the last excitation pulse. Therefore

$$
p_{r}=\hbar k_{1}
$$


and as shown in the band labelled $t=\tau_{+}$we are left with a first excited state recoiling with momentum $\hbar k_{1}$ toward the ground state that is at rest. At time $t=\left(\Omega_{2} / \Omega_{1}\right) \tau$, the first excited state comes into overlap with the ground state and an echo photon having frequency $\Omega_{1}$ can be emitted toward the right. This is the tri-level echo signal.

Figure $1 \mathrm{~b}$, which gives a series of stop-action photos of a tri-level echo in the making, can be represented very concisely by a recoil diagram such as is presented in Figure 1c. ${ }^{10}$ Here we have plotted the recoil positions of the various states leading to the tri-level echo as a function of time. The ground state trajectory is represented as the solid line along the time axis. The first and second excited states are represented by dashed and dotted lines, respectively. When two lines representing two different states intersect there is overlap and an echo may be emitted, depending on the relative momenta of the two states and the existence of a radiative moment that couples the two states. In Figure 1c the lines representing the first excited state and the ground state intersect at time $t=\left(\Omega_{2} / \Omega_{1}\right) \tau$, which is the time of the emitted tri-level echo. The wiggly lines in Figure 1c represent photons that are either absorbed or emitted. Photons are absorbed from pulse 1 and pulse 2 while pulse 3 stimulates a photon to be emitted. We have also drawn an echo photon being emitted. This feature of Figure 1c emphasizes that the tri-level echo is in fact a type of delayed four-wave mixing. ${ }^{11}$

\section{ANGLED BEAM ECHOES}

We now analyze an ordinary two-pulse echo experiment performed with angled excitation pulses. Let $\mathbf{k}_{1}$ and $\mathbf{k}_{2}$ be the wave vectors of pulses 1 and 2, respectively. $\mathbf{k}_{1}$ is along the $x$-axis and $\mathbf{k}_{2}$ is at an angle $\theta(\theta \ll 1)$ with respect to $\mathbf{k}_{1}$, thus having a small component along the positive $y$-axis, as shown in Figure 2a. If we call $\tau$ the temporal separation between the excitation pulses then the sequence of events leading to the echo at time $2 \tau$ is as presented in Figure $2 b$.

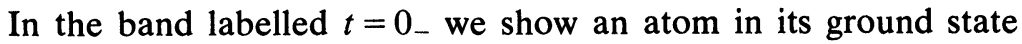
represented by the solid-line circle, and an approaching laser pulse having wave vector $\mathbf{k}_{1}=k(1,0)$ in the coordinate system introduced in Figure $2 \mathrm{~b}$. We assume that the laser pulse has area $\pi / 2$ so that after its passage the atom is in a 50-50 superposition of its ground 

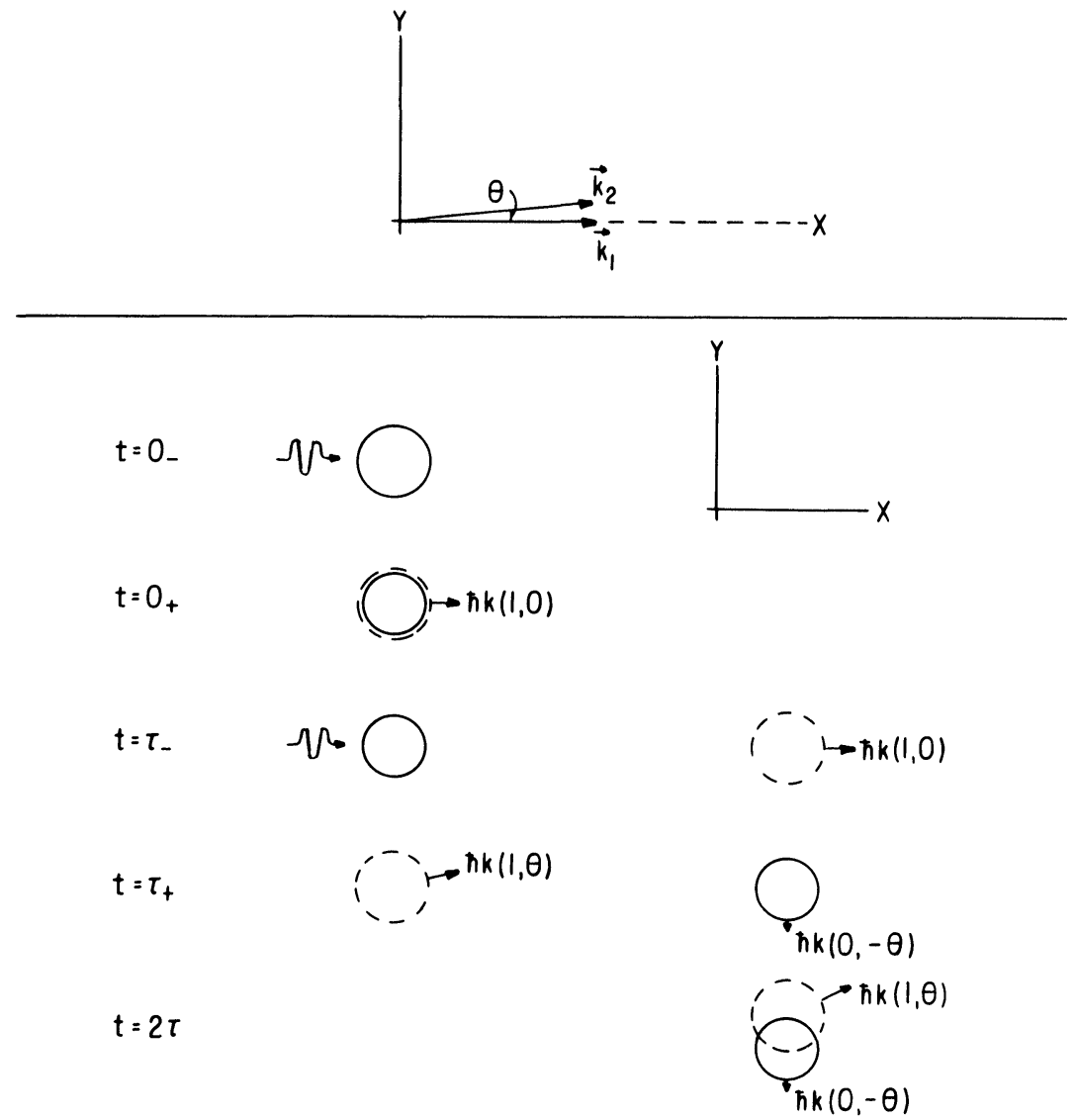

FIGURE 2 (a) Excitation pulse orientation used in a two-pulse angled-beam echo experiment. (b) The sequence of events leading to the echo. Solid line circles denote the ground state and dashed line circles denote the excited state. (c) The tracking of ground and excited states required for echo generation. (d) Recoil diagram showing the kinematics of a two-pulse angled-beam echo.

and excited states. This is shown in the band labelled $t=0_{+}$where we show the excited state, represented by the dashed-line circle, recoiling with the momentum of the absorbed photon. We now let the system develop freely until $t=\tau$ when we send in laser pulse 2 . This is depicted in the band labelled $t=\tau_{\text {- where we show the now }}$ separated ground and recoiling excited states and the approaching 


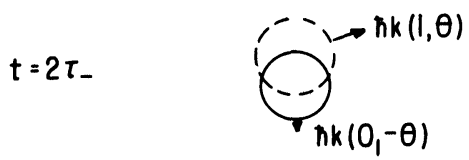

$t=2 \tau_{+}$

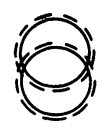

$t=3 t$

$O_{n k(0,-\theta)}$

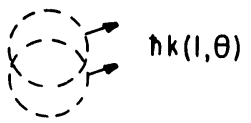

FIGURE 2(c)

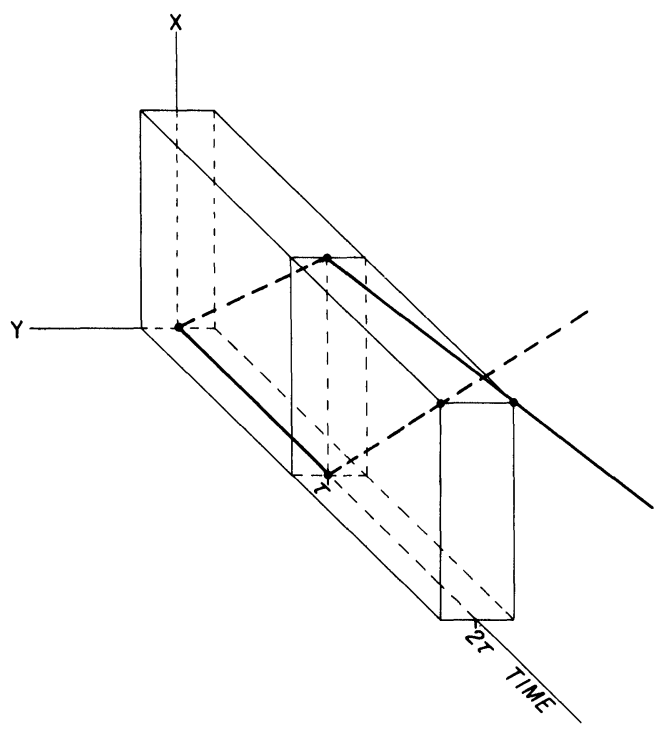

FIGURE 2(d) 
second excitation pulse. We assume pulse 2 has area $\pi$ and so has the property of transforming ground to excited and excited to ground state with $100 \%$ probability. After the passage of pulse 2 the situation is as depicted in the band labelled $t=\tau_{+}$. The newly formed excited state is recoiling with the momentum of the absorbed photon, $\hbar \mathbf{k}_{2}=$ $\hbar k(1, \theta)$, to lowest order in $\theta$. The new ground state which was formed from an excited state by being stimulated to emit a photon having momentum $\hbar k_{2}$ is itself recoiling with momentum $\hbar k(1,0)-$ $\hbar k(1, \theta)=\hbar k(0,-\theta)$ as required by momentum conservation. At time $t=2 \tau$ the ground and excited state wave packets are at their point of closest approach. If the excitation beams had not been angled there would be perfect overlap at this time. As it is the overlap has been degraded and so it can be expected that the echo amplitude will also be degraded.

We now consider the direction of the emitted echo. One approach is to require that when the superposition that exists at time $2 \tau$ interacts with the echo radiation the net effect be that the population of the ground state increases while the population of the excited state decreases. This is the only way in which an echo can be generated. In Figure $2 \mathrm{c}$ we show the superposition at time $t=2 \tau_{-}$, just before the echo is emitted by the sample. If we now look at the emitted echo radiation as an excitation pulse having small area we can say that its effect on the superposition at time $t=2 \tau_{-}$is to transform part of the excited state into a ground state and transform part of the ground state into an excited state. This is depicted in the band labelled $t=2 \tau_{+}$of Figure $2 \mathrm{c}$. But now we must require that the newly generated ground state track with the ground state that was present initially and similarly for the excited states; anything else would violate conservation of the number of atoms. If the states did not track then when they eventually became separated we could perform the following integral

$$
\int \Psi^{*}(\mathbf{r}, t) \Psi(\mathbf{r}, t) d \mathbf{r}
$$

The contribution to this integral from the initial ground and excited states, present at $t=2 \tau_{-}$, would give 1 so the added contribution from the newly generated ground and excited states would make the integral greater than 1 which is clearly impossible. In Figure 2c we show the state of the system at $t=3 \tau$ : both ground states tracking 
together and both excited states tracking together. It turns out, as can be seen by keeping track of relative phases, that the two excited states exhibit destructive interference while the two ground states exhibit constructive interference. This is the expected result: the population of the excited state has decreased while the population of the ground state has increased. To find the direction of the emitted echo is now a simple matter. Consider the new excited state, generated from the ground state present at $t=2 \tau_{-}$by interacting with the echo radiation. The momentum of this new excited state must equal the momentum of the original excited state since we require they track together. Therefore the momentum of the photon that is absorbed by the ground state to generate this new excited state must be given by

$$
\hbar k(1, \theta)-\hbar k(0,-\theta)=\hbar k(1,2 \theta),
$$

the momentum of the initial excited state minus the momentum of the initial ground state. This is just a restatement of the familiar result that the direction of the emitted echo, $\mathbf{k}_{\text {echo, }}$, is given in terms of the wave vectors of the excitiation pulses by ${ }^{2}$

$$
\mathbf{k}_{\text {echo }}=2 \mathbf{k}_{2}-\mathbf{k}_{1} \text {. }
$$

The same result can be arrived at by considering the stimulated emission of a photon from the original excited state present at $2 \tau_{-}$ to generate the new ground state.

As was the case in our analysis of the tri-level echo we can represent the events leading to an echo in the present case in a recoil diagram. This is presented in Figure 2d. Since we now need two spatial axes to represent the trajectories and one time axis the recoil diagram becomes three dimensional. The trajectory of the ground state is represented by the solid line and the trajectory of the excited state is represented by the dashed line. As can be seen in the diagram the point of closest approach between the ground and excited state wave packets occurs at $t=2 \tau$, the time of the echo.

We have already shown that when using angled excitation pulses the echo amplitude will be degraded from what it would have been had parallel excitation pulses been used. Nevertheless, there are situations when performing an echo experiment in which it is advantageous to use angled excitation beams, the biggest advantage being that when using angled excitation beams in a large Fresnel-number 
sample it becomes possible to separate the echo from the excitation pulses using passive component such as a lens and a pinhole. ${ }^{12}$

Angled excitation pulses can also be used to probe atomic velocity distributions. In the experiment just analyzed this is not very interesting since the velocity distribution is well known to be Maxwellian. But one can imagine situations with transient velocity distributions which can be monitored with this technique.

\section{OBSERVATION OF ANGLED BEAM ECHOES}

To demonstrate this technique and also the large dynamic range over which echo signals can be observed we have recently performed a two-pulse echo experiment on the $2^{2} S_{1 / 2}-2^{2} P_{3 / 2}$ transition in lithium vapor. ${ }^{12}$ This experiment was performed with angles of $0.70 \pm$ $0.06 \mathrm{mrad}$ and $1.40 \pm 0.06 \mathrm{mrad}$ between the excitation pulses. For

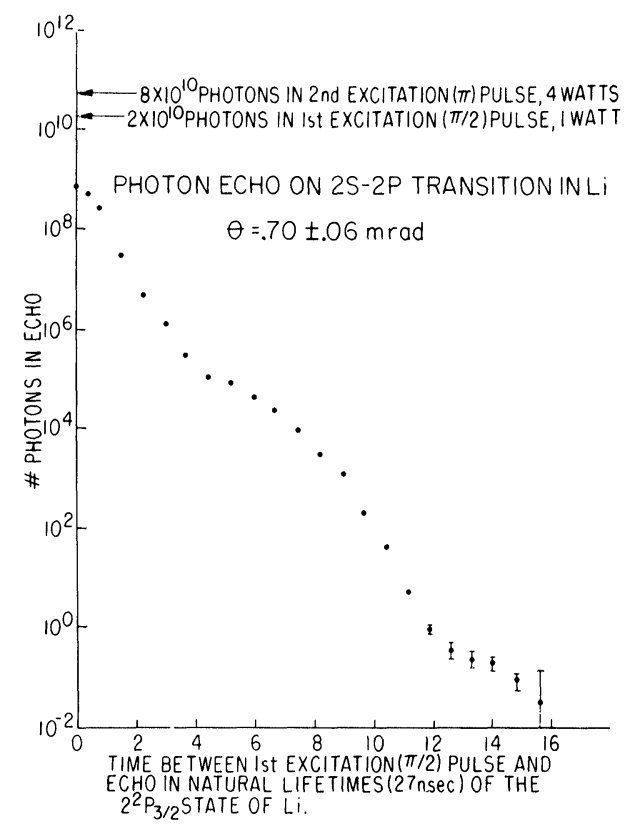

FIGURE 3 Number of photons in echo vs the separation between pulse 1 and echo in units of the natural lifetime of the $2^{2} P_{3 / 2}$ state of lithium. The angle between $\mathbf{k}_{1}$ and $\mathbf{k}_{2}$ is $0.70 \pm 0.06 \mathrm{mrad}$. 
each angle the pulse separation was swept from $5 \mathrm{~ns}$, until even counting 200 echoes we could no longer detect a signal. Our data is shown in Figures 3 and 4 . The data taken with an angle of $0.70 \mathrm{mrad}$ between the excitation pulses covers an intensity range of more than $10^{10}$ allowing the echo decay to be recorded for more than 15 natural lifetime of the $2^{2} P_{3 / 2}$ state of lithium. In Figure 5 we have plotted the ratio of the data taken with $\theta_{1}=1.40 \mathrm{mrad}$ to the data taken with $\theta_{2}=0.70 \mathrm{mrad}$. As shown in Ref. 12 this ratio is

$$
R(\tau)=R_{0} e^{-2\left(\hbar q_{0} k / m\right)^{2}\left(\theta \theta_{1}^{2}-\theta \frac{z}{2}\right) \tau^{2}}
$$

where $\hbar q_{0}$ is the Doppler spread of momentum, given by

$$
\hbar q_{0}=\left(2 m k_{B} T\right)^{1 / 2}
$$

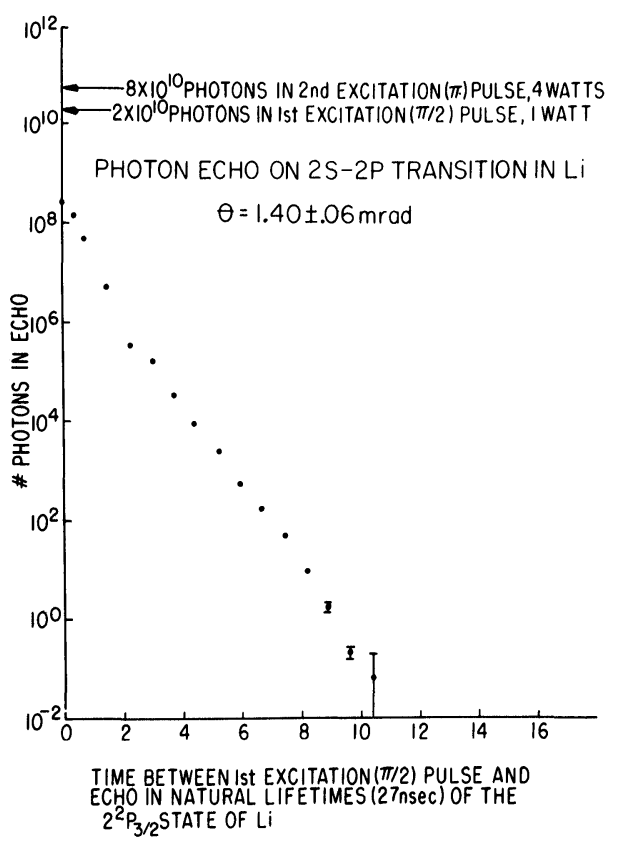

FIGURE 4 Number of photons in echo vs the separation between pulse 1 and echo in units of the natural lifetime of the $2^{2} P_{3 / 2}$ state of lithium. The angle between $\mathbf{k}_{1}$ and $k_{2}$ is $1.40 \pm 0.06 \mathrm{mrad}$. 


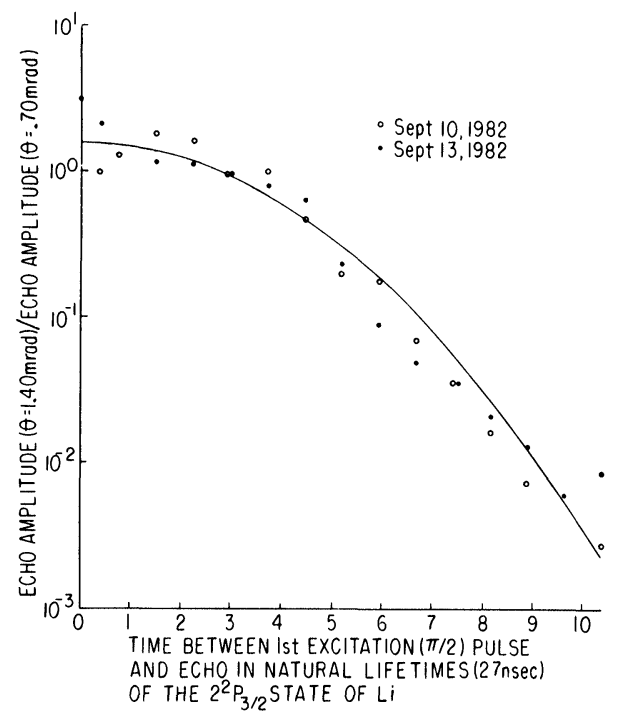

FIGURE 5 The ratio of the data plotted in Figure 4 to that plotted in Figure 3 is labelled with open dots. This ratio is also plotted for an independent run (done at the same angling of the beams) and is denoted by the solid dots. The solid line is the prediction of Eq. (39).

In Figure 5 we have also plotted $R(\tau)$. Using values relevant to the experiment,

$$
\begin{aligned}
T & =543 K \\
\lambda & =6710 \AA \\
m & =1.17 \times 10^{-26} \mathrm{~kg}
\end{aligned}
$$

$R(\tau)$ works out to be

$$
R(\tau)=R_{0} e^{-\left(\tau / \tau_{\text {eff }}\right)^{2}}
$$

where

$$
\tau_{\text {eff }}=55 \mathrm{~ns}
$$

Figure 3 demonstrates another fact that is not always appreciated in echo experiments: the maximum number of photons in the echo scales with the number of photons in the excitation pulses and can be within a factor of 10 of the $\pi / 2$ excitation pulse. ${ }^{13}$ For the data 
in Figure 3 the biggest echo is decreased from the $\pi / 2$ excitation pulse by a factor of roughly 25 . However, we have recently performed an experiment in sodium vapor in which the maximum echo was down from the excitation pulse by a factor of only 10 .

The fact that the number of photons in the echo scales with the number of photons in the excitation pulses leads to the paradoxical result that atoms having smaller oscillator strengths give rise to larger echo signals than atoms having larger oscillator strength.

\section{References}

1. E. L. Hahn, Phys. Rev.'80, 580 (1950); Phys. Today 11, 4 (1953).

2. I. D. Abella, N. A. Kurnit and S. R. Hartmann, Phys. Rev. 141, 391 (1966).

3. C. K. N. Patel and R. E. Slusher, Phys. Rev. Lett. 20, 1087 (1968).

4. M. O. Scully, M. J. Stephen and D. C. Burnham, Phys. Rev. 171, 213 (1968).

5. R. Beach, S. R. Hartmann and R. Friedberg, Phys. Rev. A. 25, 2658 (1982).

6. R. Beach, B. Brody and S. R. Hartmann, Phys. Rev. A (May, 1983).

7. A. Flusberg, R. Kachru, T. Mossberg and S. R. Hartmann, Phys. Rev. A. 19, 1607 (1979).

8. R. Kachru, T. W. Mossberg and S. R. Hartmann, Phys. Rev. A 21, 1124 (1980).

9. R. Kachru, T. W. Mossberg and S. R. Hartmann, Phys. Rev. A. 22, 1953 (1980).

10. For an interpretation of this diagram in terms of a phase dependent velocity see: T. W. Mossberg and S. R. Hartmann, Phys. Rev. A. 23, 1271 (1981).

11. P. Ye and Y. R. Shen Phys. Rev. A. 25, 2183 (1982).

12. R. Beach, B. Brody and S. R. Hartmann, Phys. Rev. A (June, 1983).

13. T. W. Mossberg, R. Kachru, S. R. Hartmann and A. M. Flusberg, Phys. Rev. A. 20, 1976 (1979). 